\title{
Mesoporous Silica
}

\author{
Yuko Ueno \\ NTT Basic Research Laboratories, NTT Corporation, 3-1 Morinosato Wakamiya, Atsugi, Kanagawa 243-0198, \\ Japan
}

Mesoporous silica (MS), which provides highly ordered, scalefine tunable mesopores (pore diameter of from 2 to $50 \mathrm{~nm}$ ) and nanopores (pore diameter smaller than $2 \mathrm{~nm}$ ), has excellent properties. These include an extremely large surface area $\left(\sim 1000 \mathrm{~m}^{2} / \mathrm{g}\right)$, high thermal stability $\left(\sim 900^{\circ} \mathrm{C}\right)$, and transparency to visible light. ${ }^{1}$ The most significant property of MS concerns its controllable and uniform porosity, because the utility of porous materials is largely due to the structure and size of their pores. Highly ordered porous structures are synthesized using a surfactant, such as block copolymers, as a template for pores that form a periodic structure in a self-organizing manner in a solution. ${ }^{2,3}$ It has been about thirty years since Yanagisawa et al. first reported the synthesis of MS in 1990 (pore diameter of from 1.8 to $3.2 \mathrm{~nm}),{ }^{3}$ but it has still attracted considerable interest in many different fields due to its great potential for wide ranging applications. These include separation, catalytic processes, molecular supports, chemical reaction fields, adsorbents, and sensors. For these applications, it is significant to understand any difference in the molecular behaviors between in pores and in the bulk phase. MS is synthesized in various forms: powder, thin-films, fibers, rods, monoliths, and spheres. Recently, Ohta et al. reported on a pore-size effect on a formation of pharmaceutical crystals from solution incorporated in the pores of MS by using Raman spectroscopy. ${ }^{5}$ Because MS exhibits an excellent transmittance in the visible wavelength region, it enables us to perform optical measurements of molecules in the pores of MS. Sato et al. reported on the diffusion of fluorescent dye in a micrometer-sized spherical MS particle by using confocal fluorescence micro spectroscopy.

(a)

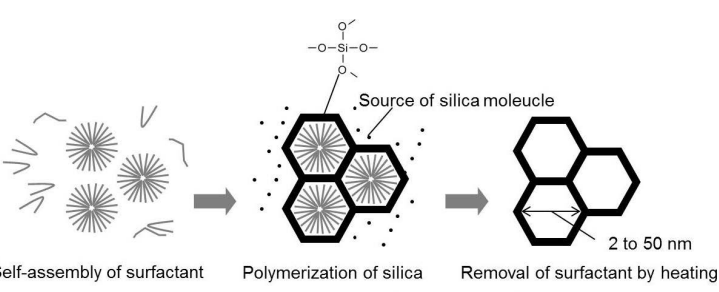

(b)

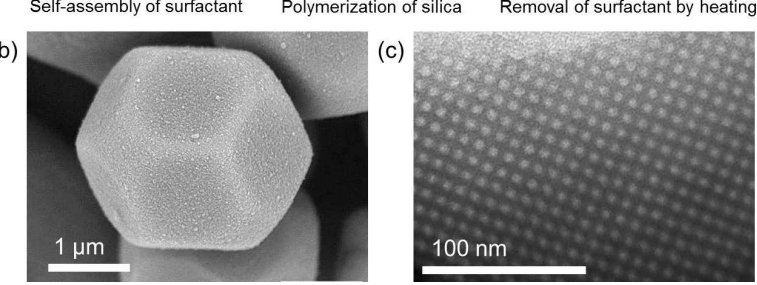

Fig. 1 Synthesis of MS using a surfactant (a), SEM (b) and TEM (c) images of SBA-16.

E-mail: ueno.yuko@lab.ntt.co.jp
The results revealed that the intraparticle diffusion of the dye is governed by the diffusion in the pore solution, which is different from those in a bulk solution. ${ }^{6}$ Fukatsu et al. reported on the size and temperature effects concerning the structures and motions of water confined in silica pores in the 1 to $10 \mathrm{~nm}$-scale range using solution-based NMR spectroscopy. The results indicated that the hydrogen-bond structures and molecular motion of water in the pores have a different dependence on the pore sizes. ${ }^{7}$ In 1998, Zhao et al. synthesized MS by using a commercially obtained triblock copolymer as a template agent for the preparation of periodic porous structured MS with larger pores (from 2 to $50 \mathrm{~nm}$ ). ${ }^{8}$ Compared with zeolites, the MS with large pores has a great advantage for incorporating molecules or functional groups into the pores. Yamaguchi et al. reported on a study concerning the adsorption behaviors of myoglobin in the pores of MS, whose pore diameters (3.9 and $6.4 \mathrm{~nm}$ ) are close to the size of a globular myoglobin $(3.52 \mathrm{~nm})$, by using differential scanning calorimetry (DSC). The results indicated that the adsorption of myoglobin into the pores of MS induced significant changes in the positions and areas of the freezing/ melting DSC peaks of the water. ${ }^{9}$ The surface structure of assynthesized MS consists of amorphous $\mathrm{SiO}_{2}$; also the chemical functions of MS, such as selective adsorption of molecules and catalytic effects are poor. Fortunately, it is easy to modify the $\mathrm{SiO}_{2}$ surface of MS pores with functional molecules, such as biological molecules and/or organic groups. Different applications become possible by using functionalized MS. Dolatyari et al. demonstrated a solid-phase extraction of uranium(VI) from water samples by using MS functionalized with a Schiff-base ligand. A high adsorption capacity with significant selectivity to uranium(VI) was successfully achieved. ${ }^{10}$ Arafune et al. reported on the adsorption dynamics of different molecular size of heme proteins, namely, cytochrome $c$ $(<3.3 \mathrm{~nm})$, myoglobin $(<3.5 \mathrm{~nm})$, and horse radish peroxidase $(<6.8 \mathrm{~nm})$ with different pore sizes of functionalized MS films ( 6.0 and $9.2 \mathrm{~nm}$ ) by using nanoporous waveguide spectroscopy. The results showed that the diffusion coefficient for heme proteins highly depended on the size and the surface functional groups. ${ }^{11}$ In this way, MS allows us to accurately quantify the molecular behaviors confined in pores with the same order of the molecular size. Application to analytical chemistry using MS can be expected to greatly expand in the future.

Keywords Mesoporous materials, mesoporous silica, adsorption

\section{References}

1. L. Bonneviot F. Béland C. Danumah S. Giasson, and S. Kaliaguine (ed.), "Mesoporous Molecular Sieves 1998", Studies in Surface Science and Catalysis, 1998, Vol. 117, 
Elsevier.

2. C. T. Kresge, M. E. Leonowicz, W. J. Roth, J. C. Vartuli, and J. S. Beck, Nature, 1992, 359, 710.

3. S. Inagaki, Y. Fukushima, and K. Kuroda, J. Chem. Soc., Chem. Commun., 1993, 8, 680.

4. T. Yanagisawa, T. Shimizu, K. Kuroda, and C. Kato, Bull. Chem. Soc. Jpn., 1990, 63, 988.

5. R. Ohta, Y. Ueno, and K. Ajito, Anal. Sci., 2017, 33, 47.

6. T. Sato and K. Nakanishi, Anal. Sci., 2017, 33, 179.

7. Y. Fukatsu, K. Morisawa, Y. Ikeda, and T. Tsukahara, Anal.
Sci., 2017, 33, 903.

8. D. Zhao, J. Feng, Q. Huo, N. Melosh, G. H. Fredrickson, B. F. Chmelka, and G. D. Stucky, Science, 1998, 279, 548.

9. A. Yamaguchi, K. Taki, J. Kijima, Y. Edanami, and Y.Shibuya, Anal. Sci., 2018, 34, 1393.

10. L. Dolatyari, M. R. Yafitan, S. Rostamnta, and M. S. Seyeddorraji, Anal. Sci., 2017, 33, 769.

11. H. Arafune, K. Hotta, T. Itoh, N. Teramae, and A. Yamaguchi, Anal. Sci., 2017, 33, 473. 